\title{
Deformation prediction analysis of vertical displacement of deep foundation pit based on LIBSVM
}

\author{
Yueyao Zhao ${ }^{1, *}$, Jiawei Zhang ${ }^{1}$, and Haojie $\mathrm{Li}^{1}$ \\ ${ }^{1}$ Shandong University of Science and Technology, College of Geomatics, 266590 Qingdao, China
}

\begin{abstract}
The reliable prediction of the surface vertical displacement deformation of deep foundation pits is of great significance to the excavation of large foundation pits. The support vector machine model (LIBSVM) has become a hot spot in the prediction of deep foundation pit deformation and provides a new prediction for the deformation of deep foundation pits. In this paper, taking the deep foundation pit of Daoxianghu Road Station in xx as an example, a prediction model of vertical displacement on the ground is established based on LIBSVM and analysis shows that the prediction results based on the model are in good agreement with the measured data, and the MSE reaches 0.0323 . The model is effective and has an effective prospective skill.
\end{abstract}

\section{Introduction}

With the rapid development of economy and science and technology, the deformation prediction of deep foundation pits has become the primary task in deep foundation pit engineering. For the prediction method of vertical displacement deformation of deep foundation pits, scholars at home and abroad have carried out many corresponding studies, using time series analysis [1], gray system theory [2],genetic algorithm [3]and artificial neural network theory [4].The method is applied to the prediction of the vertical displacement deformation of deep foundation pits, and the prediction model is established to realize the deformation prediction.

However, it is not feasible in large-scale projects. In this paper, LIBSVM is used for regression prediction of surface vertical displacement data. Through comparison and analysis between the measured data and the predicted data, it is found that the LIBSVM model is reliable and can accurately predict the subsequent deformation of the foundation pit.

\section{SVM principle}

\subsection{SVM model constructions}

The support vector machine method is based on the VC dimension theory of statistical theory and the principle of minimum structural risk. According to the limited sample information, the complexity of the model (that is, the accuracy of learning of a specific training sample, Accuracy) and learning ability (that The ability to identify arbitrary samples without error) seeks the best compromise between them in order to obtain the best promotion ability [6](or generalization ability).

SVM is developed from the optimal classification surface under linearly separable conditions. The basic idea is shown in Figure 1.

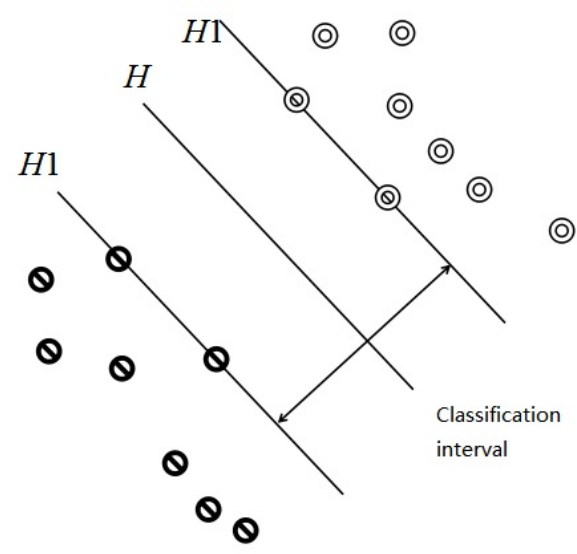

Fig. 1 Sketch Map of Optimal Classification Plane

In the figure, $\mathrm{H}$ is the classification surface, and $\mathrm{H} 1$ and $\mathrm{H} 2$ are parallel to $\mathrm{H}$. The solid points and the hollow points represent two types of samples, and the distance $\Delta$ between them is called the classification interval [3].

\subsection{Kernel functions}

The kernel function is given a function $K$, for all $\mathrm{x}, \mathrm{Z} \in \mathrm{x}$, satisfy:

$$
K(x, z)=\varphi(x) \bullet \varphi(z)
$$

(1) where $\mathrm{K}$ is the kernel function and $\varphi$ is the

\footnotetext{
* Corresponding author: skzhaoyueyao@163.com
} 
mapping function.

Two common kernel functions:

(1) Polynomial kernel function

$K(x, z)=[(x \cdot z)+c]^{p}, c \geq 0, p=1,2, \cdots$

In the formula, $\mathrm{p}$ is the order of the polynomial, and the result is the p-order polynomial classifier.

(2) Gaussian radial basis kernel function (RBF)

$$
K(x, z)=\exp \left\{-\frac{|x-z|^{2}}{\sigma}\right\}
$$

In the formula, $\sigma$ is a controllable parameter. The SVM obtained by the above formula is a radial basis classifier. The basic difference from the traditional radial basis function method is that the center of each kernel function corresponds to a support vector. The output weights are automatically determined by the algorithm [7].

SVM solves the common problems of neural networks, but in practice there will be some shortcomings of small samples and local minimums. In the actual settlement monitoring project, facing the problem of large monitoring data, the LIBSVM algorithm uses the advantages of the SMO algorithm and the SVMLIGHT algorithm to solve the shortcomings of the large amount of sample data and can be effectively predicted.

\section{Project examples}

\subsection{Monitoring data}

The data source includes the deformation monitoring data of the supporting piles at different depths during the excavation of the foundation pit. When performing data prediction, the original data should be pre-processed, so that a gross error in the data leads to a larger error in the prediction model. Select data from different time periods for analysis, and plot the trend of the cumulative settlement of all observation data with time, as showed in Figure 2 below. It can be seen from the figure below that no large jumps are found in the time series in the observation data, so replacement and interpolation are not required [8].

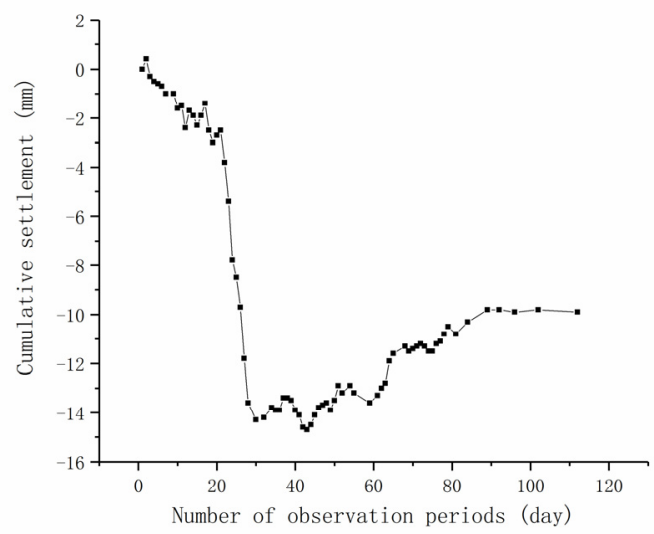

Fig. 2 change curve of cumulative settlement at point DB-14

\subsection{Comparative analyses of predicting results and measured data}

In this paper, the LIBSVM software package developed by Professor Lin Zhiren of Taiwan University is used for regression prediction of the vertical displacement data of the surface [9].

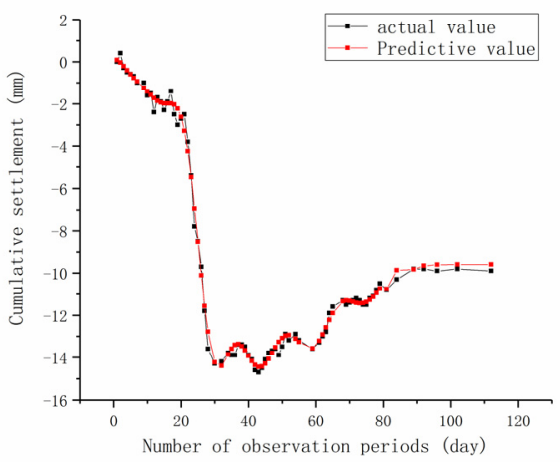

Fig.3 comparison curve of predicting value and measured value based on LIBSVM

As showed in Figure 3, regression prediction results using LIBSVM software are more reliable and the fitting accuracy is higher. However, some deviations in the predictions in the latter 10 periods are mainly due to the influence of the external environment, but they are all within the error range. The largest training fitting error is $-0.8624 \mathrm{~mm}$, the smallest training fitting error is $0.030 \mathrm{~mm}$, the largest prediction error is $-0.436 \mathrm{~mm}$, and the smallest prediction error is $0.021 \mathrm{~mm}$.

The comparison chart and the residual plot of the predicted and measured values of LIBSVM are shown in the following figure:

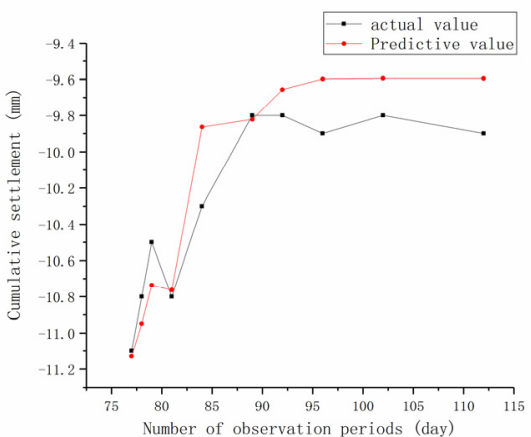

Fig.4 comparison between predicting value and measured value

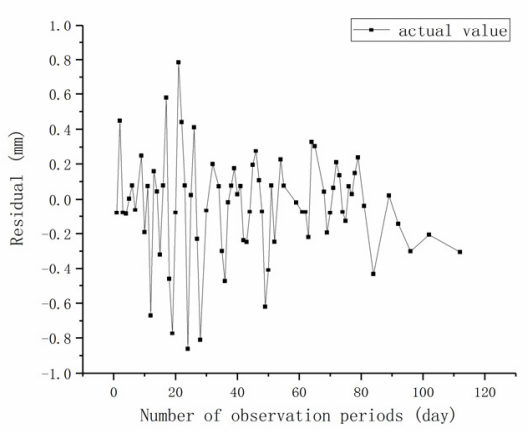

Fig.5 prediction residual diagram 
Table 1 shows the comparison between the measured value and the predicted value in the last 10 periods

Table 1 prediction results

\begin{tabular}{|c|c|c|c|c|}
\hline time & $\begin{array}{c}\text { Measured } \\
\text { value }(\mathrm{mm})\end{array}$ & $\begin{array}{l}\text { Predictive value } \\
\quad(\mathrm{mm})\end{array}$ & Residual (mm) & $\begin{aligned} \text { Set actual measured value } & y_{i}, \text { Fitted or predicted } \\
& \wedge \\
\text { value } & y_{i}\end{aligned}$ \\
\hline $12 / 25$ & -11.1000 & -11.1295 & 0.0295 & \multirow{7}{*}{$\begin{array}{l}\text { Mean absolute percentage error(MAPE) } \\
\text { MAPE }=\frac{1}{\mathrm{n}} \sum_{i=1}^{n} \frac{\left|y_{i-} \hat{y_{i}}\right|}{y_{i}}=0.0183 \\
\text { Mean square error(MSE): } \\
M S E=\sqrt{\frac{1}{\mathrm{n}} \sum_{i=1}^{n}\left(y_{i-} \hat{y_{i}}\right)^{2}}=0.0323 \mathrm{~mm} \\
\text { Mean absolute error(MAE): }\end{array}$} \\
\hline $12 / 26$ & -10.8000 & -10.9476 & 0.1476 & \\
\hline $12 / 27$ & -10.5000 & -10.7361 & 0.2361 & \\
\hline $12 / 28$ & -10.8000 & -10.7626 & -0.0374 & \\
\hline $12 / 30$ & -10.3000 & -9.864 & -0.436 & \\
\hline $1 / 2$ & -9.8000 & -9.8214 & 0.0214 & \\
\hline $1 / 7$ & -9.8000 & -9.6557 & -0.1443 & \\
\hline $1 / 10$ & -9.9000 & -9.5981 & -0.3019 & \multirow{3}{*}{$M A E=\frac{1}{\mathrm{n}} \sum_{i=1}^{n}\left|y_{i-} \hat{y}_{i}\right|=0.0183 \mathrm{~mm}$} \\
\hline $1 / 14$ & -9.8000 & -9.594 & -0.206 & \\
\hline $1 / 20$ & -9.9000 & -9.5945 & -0.3055 & \\
\hline
\end{tabular}

The prediction results can be seen, the prediction results based on LIBSVM meet the detection requirements, and the prediction results for the last 10 periods of up to one month are also very ideal, but there are some deviations, mainly due to external environmental factors. Carrying out predictive analysis, the results are not satisfactory. Therefore, it is feasible to use LIBSVM for regression prediction without considering the external conditions, which prove the reliability of LIBSVM in settlement prediction.

\section{Conclusion}

Based on the LIBSVM learning model, the existing deep foundation pit surface settlement monitoring data are used to establish a prediction model.Through carrying out model training on the monitoring data, and predicting the later surface subsidence and comparing it with the existing data, the following conclusions are obtained:

(1) The prediction results of surface subsidence displacement based on LIBSVM reflect that the model has a good short-term prediction effect, but as the monitoring time increases, the prediction result error will become larger and larger, which will eventually deviate from the actual situation and no longer have predictive value.

(2) According to the results of engineering examples, the prediction results of the monitoring model for the vertical displacement of deep foundation pits established by LIBSVM are close to the actual monitoring values of the project. This method has high application value for the deformation monitoring of deep foundation pits.

\section{References}

1. Yuan Jinrong, Zhao Fuyong. Time series analysis of foundation pit deformation prediction [J]. Journal of Civil Engineering, 2001(06): 55-59.

2. Chen Xiaobin, Zhang Jiasheng, An Guanfeng. Application of $\operatorname{GM}(1,1)$ and $\operatorname{GM}(2,1)$ models in foundation pit engineering prediction[J]. Journal of Geotechnical Engineering,2006(S1):1401-1405.

3. Zhou Haiqing, Chen Zhenghan. Object-oriented deep search genetic algorithm and its engineering application (II)_Algorithm verification and engineering application[J]. Journal of Rock Mechanics and Engineering, 2005(12): 2194-2202.

4. He Zhiyong, Zheng Wei. Deformation prediction of deep foundation pit built on BP neural network [J]. Journal of South China University of Technology (Natural Science Edition), 2008(10): 92-96.

5. Cheng Gong. Research on deformation prediction method founded on support vector machine [D]. Kunming University of Science and Technology, 2010.

6. Hou Rentao. Analysis and application research of settlement monitoring model of high-rise buildings [D]. 2013.

7. Zhai Xinde. Research on support vector machine for dam deformation prediction [D]. Hefei University of Technology, Geodesy and Surveying Engineering. 2007

8. Tong Guogong, Zhu Xueming, Cao Feng. Comparison[J]. Bulletin of Surveying and Mapping, 2016 (S2): 164-166.

9. Xu Zhihai, Zhang May, Li Bin, Jian Biao, Zuo 
Jianmin, Wang Yanzhi. Prediction of foundation pit monitoring data built on SVM model[J]. Engineering Construction and Design, 2019(13):186-190.

10. Francis E.H Tay,Lijuan Cao. Application of support vector machines in financial time series forecasting[J]. Omega,2001,29(4).

11. Ma Yulong. Discussion on the monitoring scheme of profound foundation pits [J]. Beijing Surveying and Mapping, 2016(01): 144-147+138.

12. Liu Zhe.My deformation monitoring and prediction based on D-InSAR and GM(1,1) model[J].Beijing Surveying and Mapping, 2018,32(04):442-446. 